\title{
Novel cardiac manifestation of USH2A and GJB2 genes: A case report
}

Mohammad Nikoo ${ }^{1}$, Mohammad Reza Hatamnejad ${ }^{1}$, Zahra Emkanjoo ${ }^{2}$, Alireza

Arjangzadeh $^{1}$, Mehdi Motahari moadab ${ }^{1}$, Mehdi Bazrafshan ${ }^{1}$, and Hamed Bazrafshan ${ }^{1}$

${ }^{1}$ Shiraz University of Medical Sciences

${ }^{2}$ Rajaie Cardiovascular Medical and Research Center

September 24, 2021

\begin{abstract}
Electrical storm can be caused by structural heart diseases and/or functional electrical abnormalities. We report a young boy without cardiac risk factors, having a positive family history of sudden cardiac death who presented with electrical storm. Stepwise diagnostic approach was not fruitful to determine previously known causes as the origin of the electrical storm. A new genotype related to the current electrical storm has been found, and intracardiac pacing ceased it.
\end{abstract}

Novel cardiac manifestation of USH2A and GJB2 genes: A case report

Mohammad Hossein Nikoo, MD a

Mohammad Reza Hatamnejad, MD, MPH ${ }^{\mathrm{e}}$

Zahra Emkanjoo, MD b

Alireza Arjangzadeh, MD d

Mehdi Motahari moadab, MD ${ }^{\mathrm{d}}$

Mehdi Bazrafshan, MD, MPH ${ }^{\mathrm{e}}$

Hamed Bazrafshan, MD ${ }^{c}$

a Associate Professor of Clinical Cardiac Electrophysiology, Shiraz University of medical science, Noncommunicable diseases research center, Shiraz, Iran

b Professor of Clinical Cardiac Electrophysiology, Rajaie Cardiovascular, Medical and Research Center, Tehran, Iran

${ }^{c}$ Associate Professor of Cardiology, Department of Cardiology Medicine, Shiraz University of medical science, Shiraz, Iran

d Department of Cardiology Medicine, Shiraz University of Medical Sciences, Shiraz, Iran

${ }^{\text {e }}$ Faculty of Medicine, Shiraz University of Medical Sciences, Shiraz, Iran

\section{Short title:}

cardiac manifestation of USH2A and GJB2

Keywords : 
Electrical storm; Sudden cardiac death; Ventricular arrhythmia; USH2A and GJB2 genes

Word Count: 1563

Study type: Case Report

Address for correspondence : Dr. Hamed Bazrafshan, Al-Zahra Charity Hospital, Department of Cardiology Medicine, Shiraz University of Medical Sciences, and Zand St, Shiraz, Iran

PO Box: 71348-14336. Tel: +989177120900. E-mail address: hamedbazrafshan@yahoo.com

\section{Key points:}

Question: What would be your next step in the approach to electrical storm when all the work-ups for structural heart disease and analysis of previous ion-channelopathies gene mutations are not significant?Findings: The result of our case report illustrates that USH2A and GJB2 genes mutation can be novel genetic causes of the electrical storm, and they should be considered for genetic analysis in a stepwise diagnostic approach.Meaning: A novel genotype responsible for the electrical storm has been reported for the first time in research.

\section{Abstract}

Electrical storm can be caused by structural heart diseases and/or functional electrical abnormalities. We report a young boy without cardiac risk factors, having a positive family history of sudden cardiac death who presented with electrical storm. Stepwise diagnostic approach was not fruitful to determine previously known causes as the origin of the electrical storm. A new genotype related to the current electrical storm has been found, and intracardiac pacing ceased it.

\section{Abbreviations list :}

VT: ventricular tachycardia

VF: ventricular fibrillation

ICD: implantable cardioverter-defibrillator

ECG: electrocardiogram

BPM: beats per minute

SCD: sudden cardiac death

TTE: transthoracic echocardiography

$\mathrm{RV}$ : right ventricular

ARVC: Arrhythmogenic right ventricular cardiomyopathy

Cx: Connexin

\section{Learning objectives}

1. To introduce USH2A and GJB2 genes mutation as possible new causes of primary genetical electrical storm

2. To demonstrate the unique presentation of this genotype by refractory ventricular storm, $\mathrm{J}$ wave in the electrocardiogram, non-significant result of echocardiography, pharmacological provocation test, and genetic analysis of previously known genes related to the electrical storm

3. To illustrate the intracardiac pacing as proper management to terminate the electrical storm caused by these novel genes

\section{Introduction}


Electrical storm is defined by three or more sustained episodes of ventricular tachycardia (VT), ventricular fibrillation (VF), or appropriate shocks from an implantable cardioverter-defibrillator (ICD) within 24 hours (1). Despite the large proportion of structural heart diseases, such as coronary artery disease, cardiomyopathies, congenital and valvular heart disease that lead to the electrical storm, it can be induced due to inherited channelopathies, electrolyte disturbances, endocrinologic abnormalities, and other secondary causes (2). In this case report, we present refractory ventricular storm in a young boy, which was not related to previously known causes of electrical storm and was so challenging to manage. Two gene mutations related to electrical storm have been found and reported for the first time.

\section{Case presentation}

On January $1^{\text {st }}, 2020$, a non-smoker 17 -year-old Iranian boy, with a previous history of migraine headaches, as the only significant past medical history, referred to a local hospital with complaints of abrupt loss of consciousness before admission. Standardized approach to syncope was done, and electrocardiogram (ECG) showed ventricular tachyarrhythmia as the possible cause of syncopal attack; thus, the patient had been transferred to our tertiary center for ICD insertion, after stabilization. On admission, his vital signs showed a blood pressure of $105 / 70 \mathrm{mmHg}$, heart rate of 120 beats per minute (BPM), respiratory rate of 22 breaths per minute, and temperature of 36.7 C. Physical examination was unremarkable. Following the ICD insertion (Figure 1), he was discharged with propranolol 10mg twice daily. After 2 weeks, he was readmitted to the hospital with discharged ICD generator due to recurrent episodes of shock releasing. Electrical storm was diagnosed regarding 16 appropriate ICD therapies for ventricular tachyarrhythmia in 24 hours. He was born from consanguineous marriage. The patient declared 2 cases of sudden cardiac death (SCD) with an unknown specific cause in his aunt and cousin (both on his father's side) at the age of 25 and 30 years, respectively; therefore, transthoracic echocardiography (TTE) was done to rule out the structural heart disease as the result of normal biventricular size and function (ejection fraction of $55 \%$ ). Functional electrical abnormalities were considered the main cause. The patient denied taking any medication or substance, and laboratory data ruled out the electrolyte and endocrinologic abnormalities (Table 1), so ion channelopathies were evaluated. Although the baseline ECG (Figure2A, B) demonstrated ' J wave ', the patient experienced episodes of torsades the point (Figure 2C-F) during the cardiac care unit course; thus, pharmacological provocation tests using procainamide and epinephrine were done respectively to diagnose $\mathrm{J}$ wave syndrome, long QT syndrome, and catecholaminergic polymorphic ventricular tachycardia. Provocative drug tests did not specify any causes of the storm. ICD reprogramming and medical treatment including amiodarone, beta-blockers, verapamil, and magnesium to control refractory VT/VF storm failed; therefore, the patient was brought to the catheterization laboratory for radiofrequency ablation. No arrhythmic focus was observed in epicardial and endocardial 3-dimensional mapping for ablation. We were forced to use deep sedation and general anesthesia to control the electrical storm. Empirical testing of the intracardiac pacing with a rate of $90 \mathrm{bpm}$ was fruitful to cease the subsequent VT storm. Controlling the electrical storm via pacing was in favor of long QT syndrome as the cause of the storm. Thus, genetic analysis to identify the related long QT syndrome genes was assessed. Surprisingly whole-exome sequencing (using Next Generation Illumina Sequencing) did not reveal any pathogenic mutations related to ion channelopathies; nevertheless, two known (bi-allelic) heterozygous mutations in USH2A (NM_206933:exon29:c.C5836T:p.R1946X) and GJB2 (NM_004004:exon2:c.G71A:p.W24X) genes were detected. Up to now, good condition without facing the previous syncope and palpitation events, and normal ECG (Figure 2G) have been documented in follow-up visits to the outpatient clinic. The university ethics committee approved the study protocol. The patient gave written informed consent.

\section{Discussion}

Our case was so challenging and had a complicated scenario. At first, rapid screening was done by 12-leads ECG, laboratory analysis, and TTE. No evidence of electrolyte disturbances, infections, hyperthyroidism, intoxication, and substance abuse was shown in laboratory parameters. TTE indicated no sign of heart failure, valvular heart disease, and wall motion abnormalities. The patient had no indications to undergo coronary angiography and revascularization due to negative history of chest pain and dyspnea, not elevated 
cardiac biomarkers, and lack of ischemic changes in ECG; also, atherosclerosis and coronary artery disease in a 17-year-old boy was rare. There was no reason to carry out cardiac magnetic resonance due to the absence of structural heart disease clues in TTE. Also, the absence of scar formation structure in epicardial and endocardial 3-dimensional mapping ruled out structural heart disease such as ARVC. Primary inherited genetic abnormalities remained as the main cause. The most confusing point about this case was that ECG, containing the normal QTc interval and $\mathrm{J}$ wave, was in favor of $\mathrm{J}$ wave syndrome, e.g. Brugada syndrome, while termination of electrical storm via intracardiac pacing was in agreement with long QT syndromes, according to the guideline (3). In contrast to all of these interpretations, pharmacological provocation test did not reveal any causes, and whole-exome sequencing revealed mutations in USH2A and GJB2 genes that were responsible for the current electrical storm. Nevertheless, no literature has announced the relationship between USH2A (responsible for type 2 Usher syndrome) and GJB2 genes and refractory electrical storm. Usher, a genetic syndrome that consists of visual impairment due to retinitis pigmentosa and varying levels of deafness, mainly caused by impairment in usherin (4). Usherin which is encoded by the USH2A gene has been recognized as a basement membrane protein in retinal photoreceptors and cochlear hair cells (5), but Bhattacharya et al. (6) announced usherin can be presented in other tissues of organs, despite not being affected in usher syndrome. Even though in his work there was no sign of usherin in cardiac tissue, however, the role of usherin in the basement membrane of myocyte can not be ruled out firmly. A recent paper elucidated the association between usher syndrome and tachyarrhythmia by claiming the myocytes' disconnection (7). Thus the defect in the cell by cell electrical propagation, a result of intercellular connection disruption, can be concluded as a consequence of their basement membrane alteration due to USH2A mutation. Direct communication among the two cells e.g. transmembrane signaling via ions exchange between adjacent cells, is mediated by gap junction. Gap junction as a channel is constructed by two docked connexons of apposing cells and each connexon consist of six connexins (an integral membrane protein) subunits. Connexin $(\mathrm{Cx})$ proteins as a crucial element for communication are expressed in different tissues. Cx43 (expressed by GJA1), Cx40 (GJA5), and Cx37 (GJA4) participate to form intercalated disk (gap junctions) in the ventricle, atrium, and vascular tree, whereas Cx 26 (GJB2) is expressed in the inner ear and is responsible for homeostasis of the cochlear fluids (8). It is known that pathogenic variants in GJB2 are the most frequently identified causes of autosomal recessive sensorineural hearing loss (9). Mutations in $\mathrm{Cx}$ genes that formed proteins of gap junction complex result in decreasing the intercellular connection and eventually leads to abnormal electrical cell by cell conductance, e.g. some patients with GJA1 mutations experienced tachycardia (8). Due to the genetic variants, the possibility of $\mathrm{Cx} 26$ presentation in the intercalated disc(gap junction) between myocytes could be supposed for the patient. Our case did not suffer from any hearing and visual impairment, so it supported our hypothesis that the expression of genes is mostly related to myocytes and their intercalated disc rather than the retinal photoreceptors and cochlear hair cells (however cardiac tissue biopsy was not applicable due to patient dissatisfaction). The most probable mechanism to justify the phenotype (electrical storm) and its genotype is combined mutation in USH2A and GJB2 change the basement membrane and intercalated disc of myocytes, and eventually leads to impairment of electrical cellular conductance. After confirmation of the presence of these genes variants in his parents, association between USH2A and GJB2 genes with electrical storm was concluded. However, the question that which gene is more important to induce electrical storm or can it be caused by each of them separately, remains unclear and more research is needed.

\section{Conclusion}

This case illustrated USH2A and GJB2 genes as novel genetic causes of the electrical storm for the first time in research; also, intracardiac pacing has been declared as the proper management to cease the ventricular storm, which is caused by this novel arrhythmic genotype. We recommend this novel genotype should be named 'BEMAN syndrome'. Further investigation is needed to determine the exact mechanism.

Acknowledgment : The authors would like to thank the participant and his family, Shiraz University of Medical Sciences, Shiraz, Iran, and Center for Development of Clinical Research of Nemazee Hospital, Dr. Nasrin Shokrpour for editorial assistance. 
Conflict of interest : The authors confirm that there are no known conflicts of interest associated with this publication and there has been no significant financial support for this work that could have influenced its outcome.

Funding: This research received no grant from any funding agency in the public, commercial or not-for-profit sectors.

Patient Consent: Written informed consent was obtained from the patients regarding the publication of this study. There is no identifying information in this article.

Ethics approval: The university ethics committee approved the study protocol and all the study procedures were conducted following the Declaration of Helsinki.

Data availability: Data of the participant can be requested from the authors. Please write to the corresponding author if you are interested in such data.

\section{References:}

1. Eifling M, Razavi M, Massumi A. The evaluation and management of electrical storm. Texas Heart Institute journal. 2011;38(2):111-21. Epub 2011/04/16. PubMed PMID: 21494516; PubMed Central PMCID: PMCPMC3066819.

2. Savastano S, Dusi V, Baldi E, Rordorf R, Sanzo A, Camporotondo R, et al. Anatomical-based percutaneous left stellate ganglion block in patients with drug-refractory electrical storm and structural heart disease: a single-centre case series. Europace : European pacing, arrhythmias, and cardiac electrophysiology : journal of the working groups on cardiac pacing, arrhythmias, and cardiac cellular electrophysiology of the European Society of Cardiology. 2021;23(4):581-6. Epub 2020/11/16. doi: 10.1093/europace/euaa319. PubMed PMID: 33190159.

3. Kowlgi GN, Cha YM. Management of ventricular electrical storm: a contemporary appraisal. Europace : European pacing, arrhythmias, and cardiac electrophysiology : journal of the working groups on cardiac pacing, arrhythmias, and cardiac cellular electrophysiology of the European Society of Cardiology. 2020;22(12):1768-80. Epub 2020/09/29. doi: 10.1093/europace/euaa232. PubMed PMID: 32984880.

4. Lenassi E, Vincent A, Li Z, Saihan Z, Coffey AJ, Steele-Stallard HB, et al. A detailed clinical and molecular survey of subjects with nonsyndromic USH2A retinopathy reveals an allelic hierarchy of diseasecausing variants. European Journal of Human Genetics. 2015;23(10):1318-27. doi: 10.1038/ejhg.2014.283.

5. Liu X, Bulgakov OV, Darrow KN, Pawlyk B, Adamian M, Liberman MC, et al. Usherin is required for maintenance of retinal photoreceptors and normal development of cochlear hair cells. Proceedings of the National Academy of Sciences of the United States of America. 2007;104(11):4413-8. Epub 2007/03/16. doi: 10.1073/pnas.0610950104. PubMed PMID: 17360538; PubMed Central PMCID: PMCPMC1838616.

6. Bhattacharya G, Miller C, Kimberling WJ, Jablonski MM, Cosgrove D. Localization and expression of usherin: a novel basement membrane protein defective in people with Usher's syndrome type IIa. Hearing research. 2002;163(1-2):1-11. Epub 2002/01/15. doi: 10.1016/s0378-5955(01)00344-6. PubMed PMID: 11788194.

7. Frustaci A, De Luca A, Galea N, Verardo R, Guida V, Carrozzo R, et al. Novel dilated cardiomyopathy associated to Calreticulin and Myo7A gene mutation in Usher syndrome. ESC heart failure. 2021;8(3):23105. Epub 2021/04/10. doi: 10.1002/ehf2.13260. PubMed PMID: 33835720; PubMed Central PMCID: PMCPMC8120391.

8. Pfenniger A, Wohlwend A, Kwak BR. Mutations in connexin genes and disease. European journal of clinical investigation. 2011;41(1):103-16. Epub 2010/09/16. doi: 10.1111/j.1365-2362.2010.02378.x. PubMed PMID: 20840374. 
9. Shen J, Oza AM, Del Castillo I, Duzkale H, Matsunaga T, Pandya A, et al. Consensus interpretation of the p.Met34Thr and p.Val37Ile variants in GJB2 by the ClinGen Hearing Loss Expert Panel. Genetics in medicine : official journal of the American College of Medical Genetics. 2019;21(11):2442-52. Epub 2019/06/05. doi: 10.1038/s41436-019-0535-9. PubMed PMID: 31160754; PubMed Central PMCID: PMCPMC7235630.

\section{Figure legends.}

Figure 1. ICD has been inserted in the proper place.

Figure 2 . Electrocardiogram on admission indicating the J wave and normal QTc (A, B) 12-lead ECG at the onset of electrical storm (C, D) Episodes of torsades the point at another time (E, F) Final ECG after intracardiac pacing in follow-up visits $(G)$.

\section{Hosted file}

Table.docx available at https://authorea.com/users/334412/articles/538379-novel-cardiacmanifestation-of-ush2a-and-gjb2-genes-a-case-report

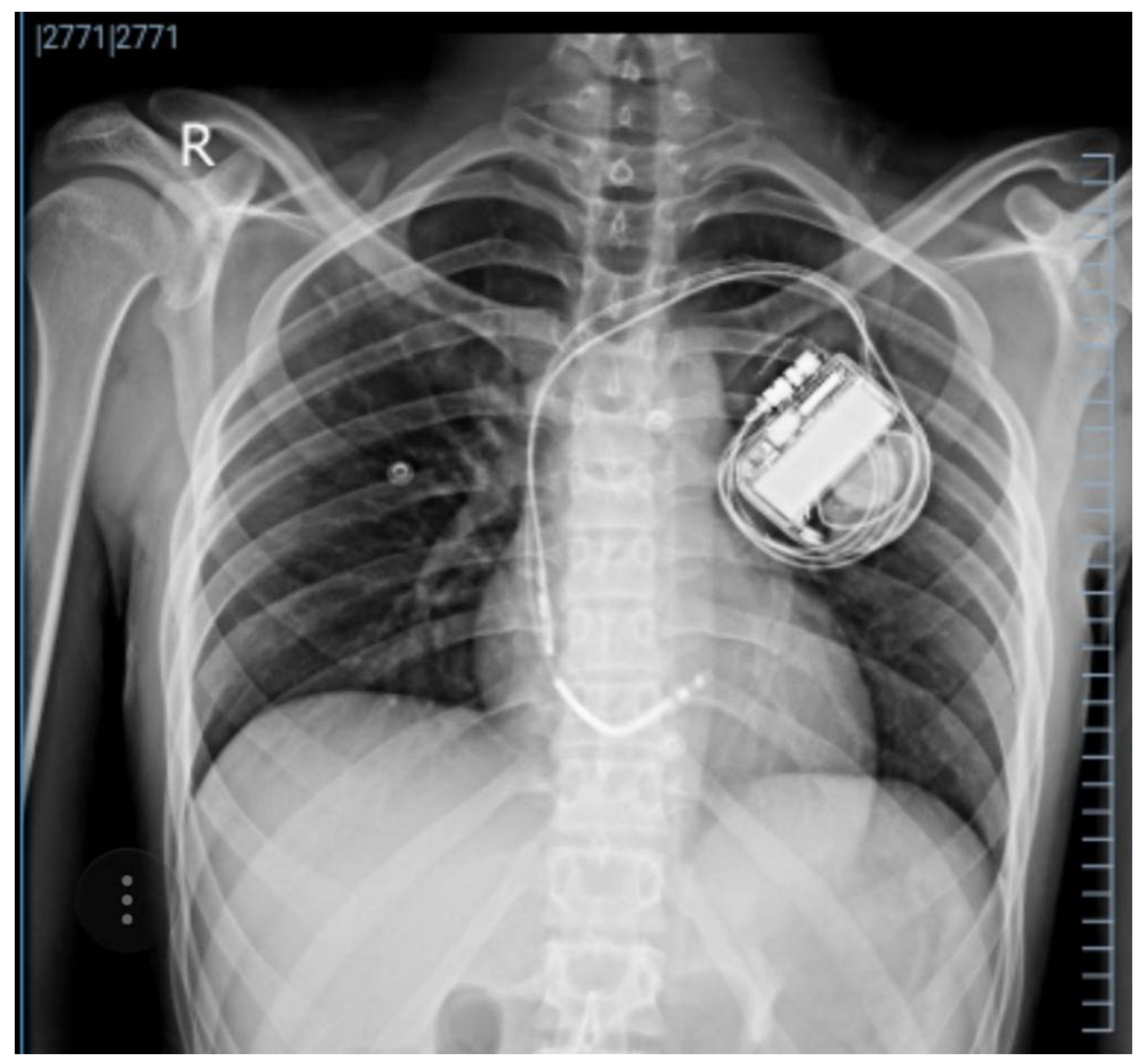




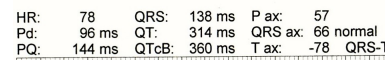


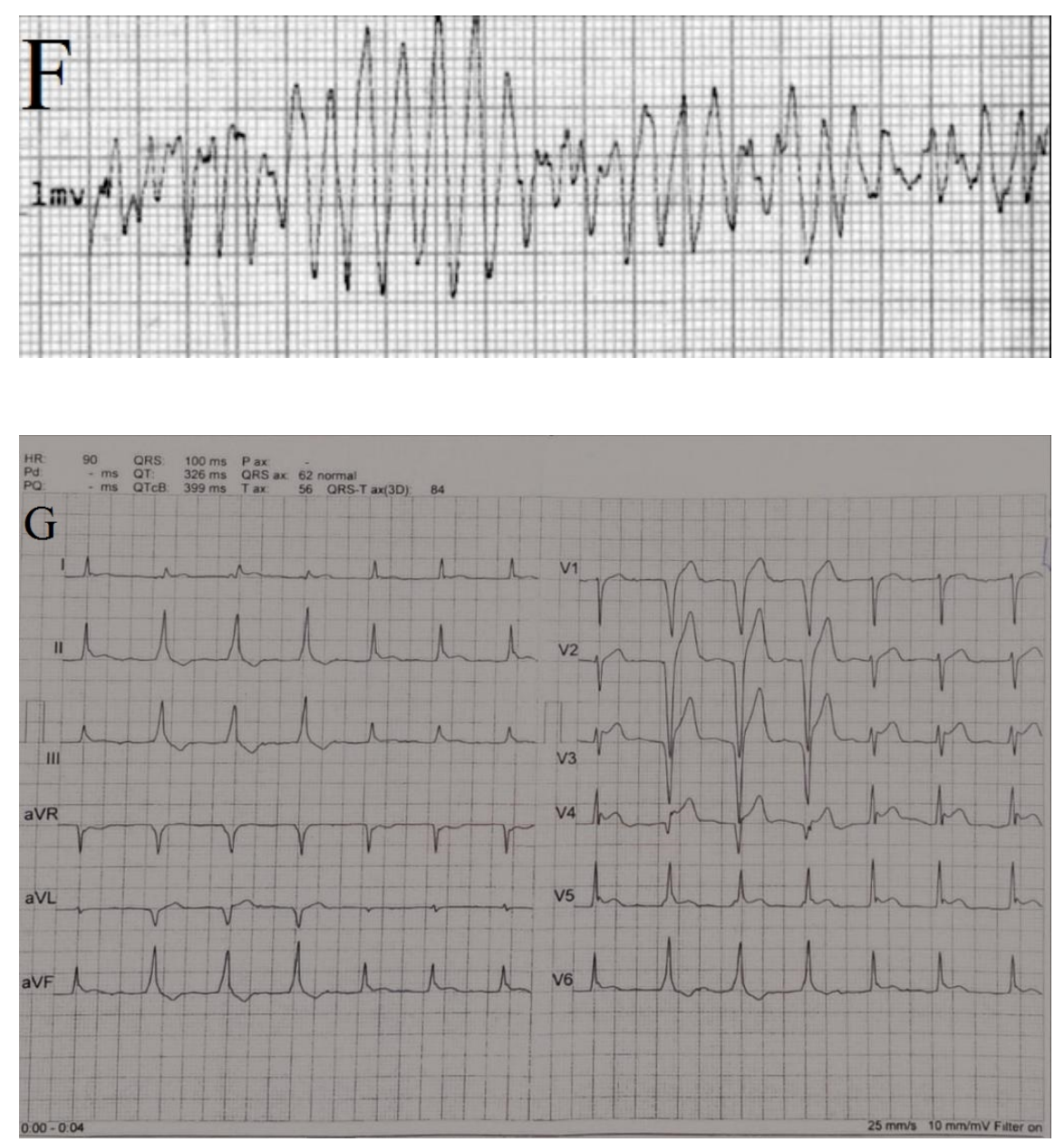